\title{
Primary Rosai-Dorfman Disease in 39-Year-Old Female with Osseous Tibial Lesion Manifestion: A Case Report and Literature Review
}

\author{
Jasmin Mansoori, DPM ${ }^{1 *}$, Ivana O Akinyeye ${ }^{2}$, Michael A Sobolevsky, DPM ${ }^{3}$ and Robert H Quinn, MD
}

\author{
${ }^{1}$ Podiatric Surgical Resident, PGY-3, Department of Orthopaedics, UT Health San Antonio, Texas, USA \\ ${ }^{2}$ Associate Podiatrist in Private Group Practice in Oxford, MS; Graduate of Podiatric Medicine and Surgery \\ Residency at UT Health San Antonio, Texas, United States \\ ${ }^{3}$ Assistant Professor and Clinical Faculty, Division of Podiatry, Department of Orthopaedics, UT Health San \\ Antonio, Texas, USA \\ ${ }^{4}$ Chair and Professor, Department of Orthopaedics, UT Health San Antonio, Texas, United States
}

*Corresponding author: Jasmin Mansoori, DPM, Podiatric Surgical Resident, PGY-3, Department of Orthopaedics, UT Health San Antonio, 7703 Floyd Curl Dr, San Antonio, Texas, USA, Tel: (469)-744-0226

\begin{abstract}
Rosai-Dorfman disease (RDD) otherwise known as sinus histiocytosis with massive lymphadenopathy (SHML), is a very rare and benign disorder of unknown etiology with < $10 \%$ bone involvement [1]. The following is a case seen at our hospital of a patient presenting with several months' onset unspecified non traumatic ankle pain. There was no physical mass or lymphadenopathy appreciated on exam. Plain radiographs and MRI demonstrated an osteolytic lesion at the medial malleolus. Biopsy revealed the diagnosis of intraosseous manifestation of Rosai-Dorfman disease.
\end{abstract}

\section{Introduction}

Rosai-Dorfman disease is a very rare and benign disorder of unknown etiology. Almost half of the cases seen have extranodal involvement (skin, soft tissue, central nervous system). Bony involvement is typically seen in $<10 \%$ of cases however primary bone RDD even less common [1]. Officially classified in 1969, this disease typically affects children and young adults with symptoms involving fever, malaise, non-painful cervical lymphadenopathy, weight loss, and pharyngitis $[1,2]$. Laboratory results will typically show elevated erythrocyte sedimentation rate or leucocytosis [1]. RDD bone lesions typically are multifocal and lytic within the cancellous areas of long bones [3,4].

The following is a case seen at our hospital presenting with several months' onset unspecified non traumatic ankle pain. Biopsy results revealed the diagnosis of intraosseous manifestation of Rosai-Dorfman disease.

\section{Case}

39-year-old Hispanic female presented with a traumatic nonspecific right ankle pain that had been progressively getting worse over the past eight months. Pain was worse at night. She related no other symptoms. Physical examination demonstrated limited and painful range of motion of right ankle. There were no skin lesions, masses, or lymphadenopathy noted. Patient had intact neurovascular status. Clinical diagnosis at the time was posterior tibial tendonitis. Pain was noted to be recalcitrant to treatment consisting of ankle brace, oral steroids, and physical therapy.

Conventional radiographs demonstrated a lytic bony lesion in the right medial malleolus (Figure 1). Magnetic resonance imaging (MRI) demonstrated a 

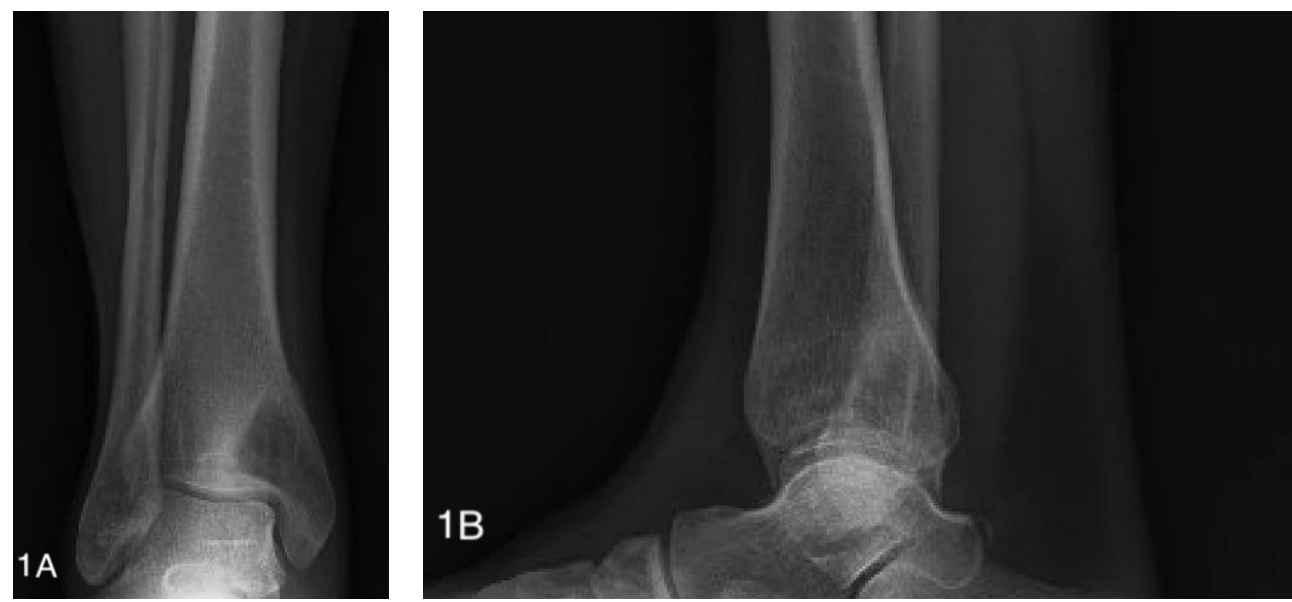

Figure 1: Conventional radiographs of the ankle reveal a lytic lesion without substantial cortical involvement. AP (a) and lateral (b) images are shown.
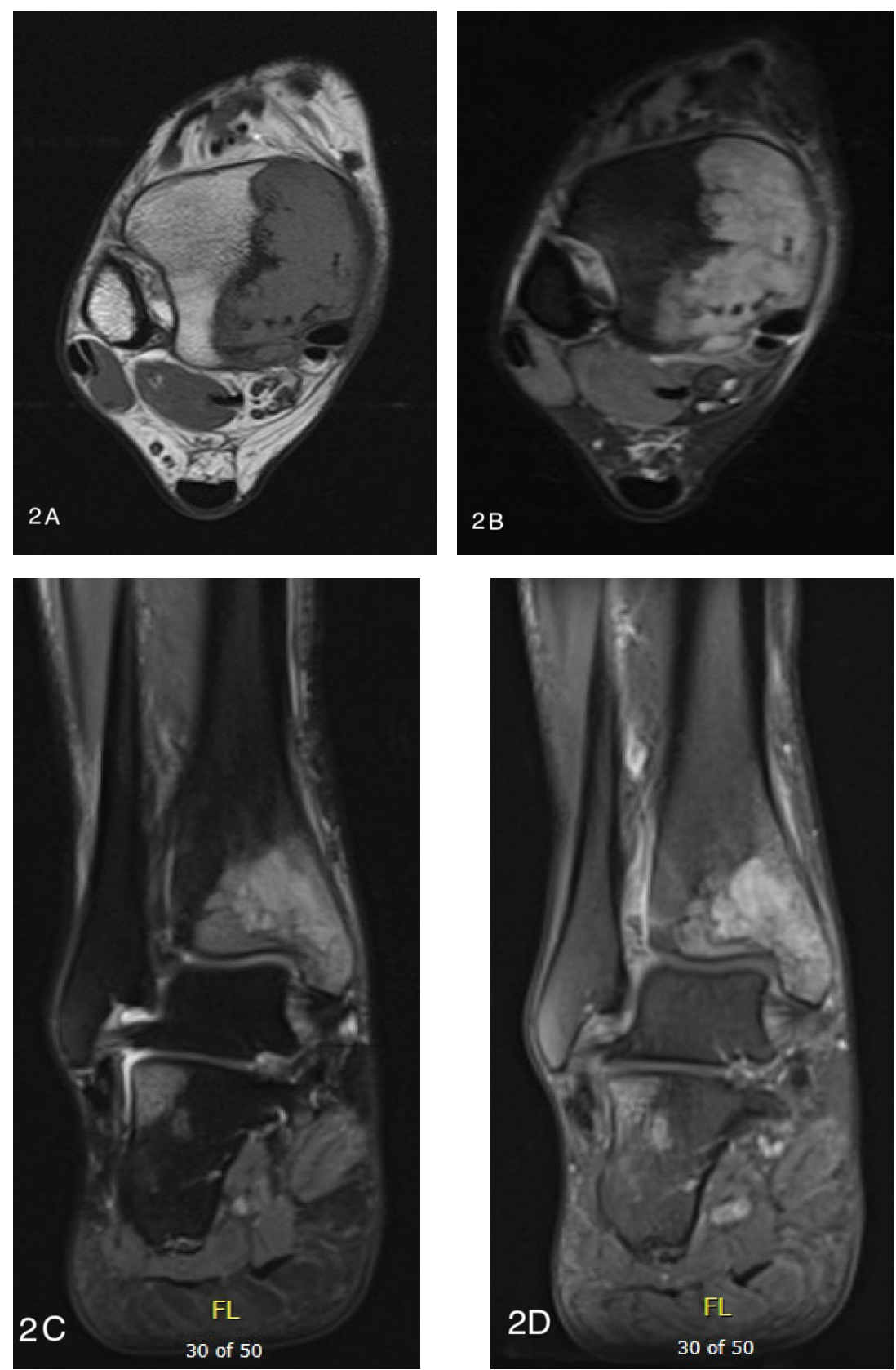

Figure 2: MRI reveals enhancing marrow replacing lesions of the medial malleolus and lateral calcaneus without significant cortical or soft tissue involvement. Axial T2 FSE (a), axial T2 FSE (b), coronal T2 FSE (c), and coronal T1 PG FS (d). 
contrast enhancing marrow replacement lesion in the medial malleolus as well as the lateral calcaneus (Figure 2). Whole body bone scan demonstrated multiple lesions throughout the bilateral lower extremites (Figure 3).

Erythrocyte Sedimentation Rate was elevated at 54. Serum $C$ reactive protein was elevated at 32.65 . White blood cell count was normal.
At that time, the patient was referred to an orthopaedic oncology surgeon on suspicion of bone tumor. CT results to determine organ involvement were negative. Needle biopsy by interventional radiology was non-diagnostic but suspicious for infection.

Open biopsy was performed by orthopaedic surgery and the patient was noted to have what was seemingly

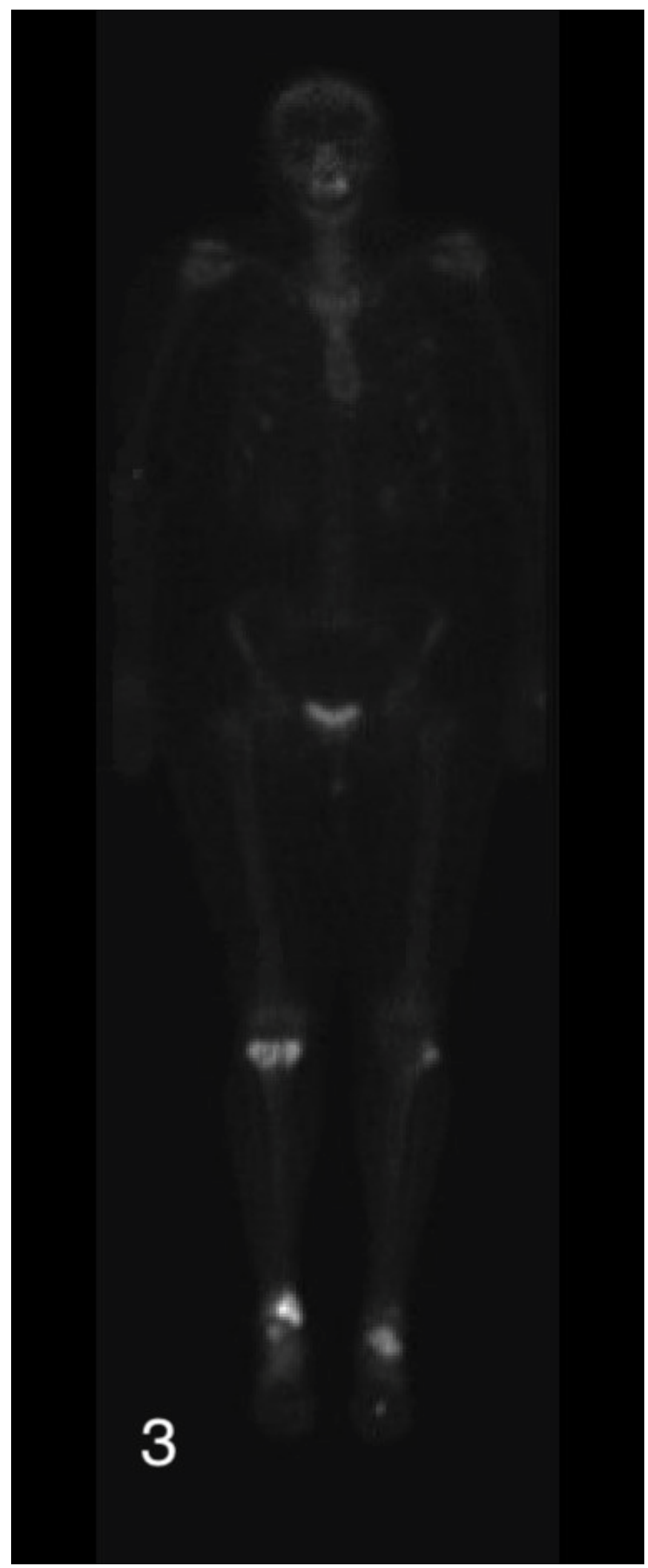

Figure 3: Whole body bone scan demonstrating multiple lesions in the bilateral lower extremities. 
purulence within cysts. Pathology results revealed the diagnosis of Rosai-Dorfman Disease. She was managed conservatively. At 6 months postoperatively her symptoms were slowly improving and her bony lesions were slowly consolidating on radiographs.

\section{Discussion}

Bone involvement occurs in $<10 \%$ cases with this disease with primary bone RDD being even more rare. More than half of the approximately 400 cases that have been registered since it's discovery in 1969 presented in extranodal sites and with constitutional symptoms [1]. To our knowledge, few other reports published have identified involvement of the foot and ankle. Osseous manifestations of RDD in the talus of patients presenting with primary musculokeletal complaints were reported by [5-7]. Most patients with RDD will have complete remission with conservative treatment. The mainstay of treatment is clinical observation [3]. Fairly recently in the literature, researchers have been documenting fine-needle aspiration cytology to diagnose osseous RDD without lymphadenopathy [8,9] although in this case even large needle biopsy was non-diagnostic. As with our patient, although isolated bone RDD is very rare, it can be a differential diagnosis in patients with musculoskeletal complaints in the foot and ankle.

\section{Conflict of Interest}

None.

\section{References}

1. Foucar E, Rosai J, Dorfman R (1990) Sinus histiocytosis with massive lymphadenopathy (Rosai-Dorfman disease): Review of the entity. Semin Diagn Pathol 7: 19-73.

2. Rosai J, Dorfman RF (1969) Sinus histiocytosis with massive lymphadenopathy. A newly recognized benign clinicopathological entity. Arch Pathol 87: 63-70.

3. Lin J, Lazarus M, Wilbur A (1996) Sinus histiocytosis with massive lymphadenopathy: MRI findings of osseous lesions. Skeletal Radiol 25: 279-282.

4. George J, Stacy G, Peabody T, Montag A (2003) RosaiDorfman disease manifesting as a solitary lesion of the radius in a 41-year-old woman. Skeletal Radiol 32: 236-239.

5. Kang RW, McGill KC, Lin J, Gitelis S (2011) Chronic ankle pain and Swelling in a 25-year-old woman: An unusual case. Clin Orthop Relat Res 469: 1517-1521.

6. Okay E, Yildiz Y, Sari T, Yildirim AN, Ozkan K (2021) RosaiDorfman Disease of the talus in a child: A case report. J Am Podiatr Med Assoc 111: 13.

7. Abdelwahab IF, Klein MJ, Springfield DS, Hermann G (2004) A solitary lesion of talus with mixed sclerotic and lytic changes: Rosai-Dorfman disease of 25 years' duration. Skeletal Radiol 33: 230-233.

8. Ruggiero $A$, Attinà $G$, Maurizi $P$, Antonino $M$, Elisabetta $T$, et al. (2006) Rosai-Dorfman disease: Two case reports and diagnostic role of fine-needle aspiration cytology. J Pediatr Hematol Oncol 28: 103-106.

9. Goel MM, Agarwal PK, Agarwal S (2003) Primary RosaiDorfman disease of bone without lymphadenopathy diagnosed by fine needle aspiration cytology. A case report. Acta Cytol 47: 1119-1122. 